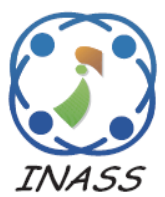

\title{
Weather and Crop Prediction Using Modified Self Organizing Map for Mysore Region
}

\author{
Pushpa Mohan $^{1 *} \quad$ Kiran Kumari Patil ${ }^{1}$ \\ ${ }^{I}$ REVA University, Computer Science Engineering, India \\ * Corresponding author's Email: pushpa.mohana@gmail.com
}

\begin{abstract}
In India Rice and ragi are the two pre-eminent crops that stable's the food of eastern and southern part of the country. These two crops mainly grown in rain fed areas, which receives heavy annual rainfall. In this scenario, Mysore district is undertaken for experiment analysis. As the region receives heavy rainfall (782mm). In this region the rainfall rate is high but the productivity of this crops are very low due to irrelevant crop cultivation. In order to overcome this concern, an advanced technology named Weighted-Self Organizing Map (W-SOM) is employed for accurate crop and weather prediction, which is the combination of both Self Organizing Map (SOM) and Learning Vector Quantization (LVQ). In this paper, the prediction accuracy is enhanced by minimizing the Within Class Error (WCE) among the clusters. Therefore, this new approach outcome shows a clear idea about suitable crop cultivation in Mysore. Experimental outcome shows that the proposed approach improved accuracy in crop and weather prediction up to $0.5-2 \%$ compared to the existing methods: SOM, Kernel-Nearest Neighbors (KNN) and Ensemble Neural Network (ENN).
\end{abstract}

Keywords: Ensemble neural network, Kernel-nearest neighbors, Learning vector quantization, Weighted-selforganizing map, Within class error.

\section{Introduction}

Agriculture is a major source for living that plays a predominant role in the development of economy all over the world. Agricultural products like food grains, edible fruits and vegetables are consumed by the people day by day [1]. Apart from this, products like tea, sugar, rice, tobacco, jute, spices etc. constitute the main items to be exported from our country to other countries. Generally, agriculture is highly dependent on the climate and rainfall condition [2]. The changes occurred in temperature, atmospheric carbon dioxide (CO2) and intensity of extreme weather could have significant impact on the crops. It affects the yield by reducing the balance in the benefits like nutrient level, moisture of the soil, water availability and frequency of blowing wind etc. [3-4]. National income and production of products are getting reduced continuously due to the sudden environmental changes like flood, drought and so on. Most of the developing countries have an average temperature, which are near or above crop tolerance level that decline 10 to $25 \%$ agricultural productivity. Individual developing countries face even larger declines. So it is necessary to analyse and forecast the climate or weather conditions frequently [5]. Indian government need to assist the farmers in cultivating crops with current climatic risks by providing valueadded weather services to every farmer. Farmers can adapt to climate changes to some degree by shifting planting dates, choosing varieties with different growth duration, or changing crop rotations.

Weather forecasting or climate prediction methods are actively raised to monitor the changes in rainfall rate [6]. Researchers are continuously developing new techniques in weather forecasting. These techniques concentrate on processing the numeric data acquired from the Mysore region. Support Vector Machine (SVM), Neural Networks (NN), K-NN, Fuzzy-C-Means (FCM), etc. are some of the common methods employed in processing of numeric data [7]. By using these conventional 
methods for clustering, a very high dimensional data is occurred. It is essential to convert the high dimensional data into low dimensional data. Generally, a lot of dimensionality reduction systems are there in crop and weather prediction to reduce "curse of dimensionality". In this paper, the SOM scheme is presented, which is a type of ArtificialNeural Network (A-NN). The SOM technique is useful for the process like abstraction, clustering and visualization of multi-dimensional data and their features [8-9]. It is also a category of unsupervised learning that needs no human interaction in detecting the data features. It provides a topology preserving mapping from a high dimensional space to map units that form a two-dimensional lattice [10]. SOM has been modified and utilized by many researchers for numerous applications related to weather forecasting.

In this research, an optimal weighting factor is added to SOM, which is generated by Learning Vector Quantization (LVQ), to improve the efficiency of crop and weather prediction. Here, the prediction accuracy is enhanced by minimizing the distance between individual weights in the cluster or reducing the WCE. Experimental outcome confirms that the advanced method shows superior improvement in prediction rate by means of accuracy, specificity, sensitivity, MAE and MAPE.

This paper is organized as follows. In Section II, the ability and issues of SOM are surveyed in Literature review section. The standards of Weighted-SOM strategy is portrayed, to seek better weather and crop prediction is proposed in Section III. In Section IV, the experimental analysis of WeightedSOM and previous algorithms are assessed. Conclusions are made in Section V.

\section{Literature review}

Several researches are suggested by researchers in crop and weather prediction. In this scenario, a brief evaluation of some important contributions to the existing literatures is presented.

P. Bose, N.K. Kasabov, L. Bruzzone, and R.N. Hartono, [11] proposed a spiking model with the knowledge of neural network for estimating the crops and their yield along with spatial-temporal analysis of the time series. The system consists of highly parallel hardware platforms with low power consuming neuromorphic characteristics. The spatial arrangement of time was accomplished from a Moderate Resolution Imaging Spectro-radiometer $250-\mathrm{m}$. The analyses on the optimum number of features were also provided to optimize the results from the experimental data set. The method predicts the yield around a month. The proposed technique delivered $95.64 \%$ of prediction accuracy. Still, more detailed studies were essential to evaluate the suitability of existing agronomic practices and alternative options for future climates. In addition, the proposed experiment was computationally expensive, especially for large datasets.

C. Lennard and G. Hegerl, [12] have developed a supervised scheme SOM for surface rainfall analysis associated to synoptic circulation. It is examined for two stations in different rainfall regimes in South Africa. This synoptic circulation was recognized for austral winter and summer as mid-latitude cyclones, whereas no circulations are related with spring and autumn rainfall. This paper evaluates the ability of SOMs to relate the synoptic drivers of observed rainfall record, which effectively downscales the large-scale synoptic information to a higher resolution surface response. This method was a relatively inexpensive downscaling tool compared to other downscaling techniques, especially regional climate models, and it was useful for assessing the effect changes in atmospheric circulation characteristics might have on a local surface response. For rainfall prediction, numerous number of attributes are required in SOM to enhance the accuracy of rainfall detection. Another major drawback of SOM was lack of an explicit projection function.

M. Capa Morocho, A.V. Ines, W.E. Baethgen, B. Rodríguez-Fonseca, E. Han, and M. Ruiz-Ramos, [13] presented an outlook model for crop yield maintenance in the region of e Iberian Peninsula. The proposed model helps to improve the security of food and economical demand of large farming. The seasonal climate forecasts were employed to process the crop forecasts. This study provides two types of ideas for merging seasonal forecasts: conditional stochastic weather generator and simple forecast probability resampler. These methods were calculated under three various seasonal rainfall methods and analyses the impacts on wheat growth after the rainfall and requirements of irrigation as well as cultivation of maize crops, which helps to quantify the benefits and risks from the seasonal weather forecasts to farmers in the Iberian Peninsula. While performing the experiment with low pressure region (Iberian Peninsula) attributes, the machine learning schemes performance was inconsistent for evaluating and analysing the impacts of crop growth.

O. Satir, and S. Berberoglu, [14] developed a crop yield estimation model using vegetation indices and stepwise linear regression model. In this literature, the related crop pattern of the area was mapped using multi-temporal landsat dataset by applying object based classification. Whereas, the prediction was 
estimated using real-time measurements like mean percent error. In this scenario, mean percent error was estimated for wheat, corn and cotton. The proposed model was only suitable for single crop prediction, for multiple crop prediction it showed limited prediction accuracy.

To overcome the above mentioned drawbacks, an unsupervised algorithm (combination of SOM and LVQ) is implemented for enhancing the performance of crop and weather prediction.

\section{Study area and site description}

In most of the developing countries, agriculture is considered as the backbone of their economy. Among the several countries, India is the largest cultivator of vegetables, fresh fruits, species, and fibrous crops like castor oil seed and jute and also the second largest cultivator of wheat and rice. In India, 60.3\% of land area is an agricultural land. The major reason for agricultural growth decline is due to water availability and post-harvest mechanism. Average rainfall rate in India is $1139 \mathrm{~mm}$ per year and most of the farmers depends on rain for cultivating the crops. In post-harvest mechanism the loss of crops is extremely high. For suitable crop cultivation and crop loss can be controlled by accurate weather and crop prediction. This paper mainly focuses on two objectives weather and crop prediction, which has numerous advantages like suitable planning of farming, whether to start harvesting or withhold and so on. The rainfall and crop cultivation is varied from place to place. Here, the Mysore region (Karnataka) is considered for data collection, because they are the largest producer of coffee, ragi, and coarse cereals and also ranked 10th in the largest rice producing state in India.

\section{Data collection and pre-processing}

In this scenario, the input data are collected from Karnataka State Department of Agriculture (KSDA) [14]. There are 12 attributes considered for crop prediction. Out of the 12 attributes, only suitable 5 attributes are chosen. Those are humidity, temperature, atmospheric pressure, soil type and rainfall. Because these are the most significant features recommended by the domain expert from KSDA. On the other hand, in weather prediction, the attributes like humidity, temperature, and atmospheric pressure are applied. In case of old data set, there is a chance for missing parameter values. Hence, pre-processing is utilized for analysis the collected data that helps to identify the absent values of the attributes. Whereas, the absent values are filled with the appropriate new values by examine the previous and past result. The pre-processed outcome data is given as the input for advanced algorithm (WSOM). The sample data collection for weather and crop prediction are mentioned in table 1 and table 2 .

Majorly three attributes are considered for weather prediction such as humidity, temperature and atmospheric pressure. In Mysore region, the temperature varies from the range of $\left(21^{\circ} \mathrm{C}-30^{\circ} \mathrm{C}\right)$, if the temperature drops the relative humidity gets increased with the range of (30 RH - $90 \mathrm{RH})$. Here, the atmospheric pressure mainly depends on three factors such as temperature, earth rotation and altitude that varies from (1007 mb-1013 mb).

Additionally, two more factors (soil type and rainfall) are under-taken to predict the suitable crop for cultivation. There are four types of soil in Mysore region such as brown forest, deep black, red soil and lateritic Soil. Based on the predicted rainfall rate and soil type, the suitable crop (either rice or ragi) is chosen for cultivation.

\section{Proposed Methodology}

This section evaluates an effective method named as standard Self Organizing Map (SOM), which is a neural network based approach for clustering and projection. In numerous researches, the SOM approach is employed as an organizer for reducing the computational cost and reproducible outcome. In this scenario, the SOM approach is utilized as a classifier in order to enhance the accuracy of prediction rate.

Table 1. Sample pre-processed data for weather prediction

\begin{tabular}{|c|c|c|c|c|}
\hline Attributes & Temperature & Humidity & Atmospheric Pressure & Class (Rainfall Prediction) \\
\hline \multirow{4}{*}{$\begin{array}{c}\text { Number of } \\
\text { collected } \\
\text { data }\end{array}$} & $f_{1}^{1}$ & $f_{1}^{2}$ & $f_{1}^{3}$ & Rainfall or no rainfall \\
\cline { 2 - 5 } & $f_{2}^{1}$ & $f_{2}^{2}$ & $f_{2}^{3}$ & Rainfall or no rainfall \\
\cline { 2 - 5 } & $f_{3}^{1}$ & $f_{3}^{2}$ & $f_{3}^{3}$ & Rainfall or no rainfall \\
\cline { 2 - 5 } & $\cdot$ & $\cdot$ & $\cdot$ & $\cdot$ \\
\cline { 2 - 5 } & $f_{k-1}^{1}$ & $f_{k-1}^{2}$ & $f_{k-1}^{3}$ & Rainfall or no rainfall \\
\cline { 2 - 5 } & $f_{k}^{1}$ & $f_{k}^{2}$ & $f_{k}^{3}$ & Rainfall or no rainfall \\
\hline
\end{tabular}


Table 2. Sample pre-processed data for crop prediction

\begin{tabular}{|c|c|c|c|c|c|c|}
\hline Attributes & Temperature & Humidity & Pressure & Soil Type & Rainfall & $\begin{array}{c}\text { Class (Crop } \\
\text { prediction) }\end{array}$ \\
\hline \multirow{4}{*}{$\begin{array}{c}\text { Number of } \\
\text { collected } \\
\text { data }\end{array}$} & $f_{1}^{1}$ & $f_{1}^{2}$ & $f_{1}^{3}$ & $f_{1}^{4}$ & $f_{1}^{5}$ & Rice or Ragi \\
\cline { 2 - 7 } & $f_{2}^{1}$ & $f_{2}^{2}$ & $f_{2}^{3}$ & $f_{2}^{4}$ & $f_{2}^{5}$ & Rice or Ragi \\
\cline { 2 - 7 } & $f_{3}^{1}$ & $f_{3}^{2}$ & $f_{3}^{3}$ & $f_{3}^{4}$ & $f_{3}^{5}$ & Rice or Ragi \\
\cline { 2 - 7 } & $\cdot$ & $\cdot$ & $\cdot$ & $\cdot$ & $\cdot$ & $\vdots$ \\
\cline { 2 - 7 } & $f_{k-1}^{1}$ & $f_{k-1}^{2}$ & $f_{k-1}^{3}$ & $f_{k-1}^{4}$ & $f_{k-1}^{5}$ & Rice or Ragi \\
\hline
\end{tabular}

In this experiment, two SOM classifiers are implemented, one classifier is employed for suitable crop prediction, and it is a month wise prediction. Another one classifier is utilized for weather prediction, which is based on day wise manner. Here, to improve the evaluation rate of two-class prediction, a weighting factor is included in the standard SOM approach that is labelled as (W-SOM).

\subsection{Weighted - Self Organizing Map (W-SOM)}

SOM is an unsupervised learning neural network algorithm, which is used to transform high dimension input signal into low dimensional discrete. SOM consists of neuron models that are systematized on a regular two dimensional grid and it has a completelyconnected network between the input layer and the neuron layer.

Initially, two input feature vectors are presented as a training sample, input vectors $x_{n}=$ $\left(x_{n 1}, x_{n 2}, x_{n 3}, \ldots x_{n m}\right)$ is applied to the input layer. The $j-t h$ unit in the competitive layer is connected to all the units on the input layer by the weight vector $W_{j}=\left(W_{j 1}, W_{j 2}, W_{j 3}, \ldots W_{j n}\right)$.

$$
Z=x_{n} \times W_{j}(t)+B
$$

Where, $Z$ is denoted as input in layers and $B$ is stated as constant bias value,

Estimated output vector value in hexagonal grid,

$$
y_{n}(p)=f(z)=f\left(x_{n} \times W_{j}(t)+B\right.
$$

In order to evaluate $y_{n}(p)$, the weight function $W_{j}(t)$ needs to be estimate. Then the Euclidean distance between the input vector and the weight of the nodes or neurons in the grid is computed. Hence, the Euclidean distance $D$ between the input vectors $x_{n}$ and the weight vector $W_{j}$ of the $j-t h$ unit in the competitive layer is determined by following equation,

$$
D\left(W_{j}, x_{n}\right)=\sqrt{\sum\left(W_{j}, x_{n}\right)^{2}}
$$

Where, $x_{n}$ is the input vector and $W_{j}$ is the random weight vector from input nodes for initializing the weight from inputs to the nodes having small random values.

The neuron having the smallest distance between input vector and weight vector is labelled as the "winner" neuron. The weights of this neuron are updated, and the distance matrix is computed iteratively for the same input vectors to minimize the error. Whereas, the unit with the minimum Euclidean distance is illustrated as the winner unit, which is represented as $j$.

Mathematically, the winner unit $j$ is stated as,

$$
j=\arg \min _{j}\left\{\left\|x_{n}-W_{j}\right\|\right\}
$$

Hence, the weight vectors of the active neuron and the neighbouring neurons are modified and updated per iteration depending on the input vector from the data set. Weight vectors of the winner unit $j$ and its neighbouring units are updated in Eq. (5),

$$
W_{j}(t+1)=W_{j}(t)+\eta(t)\left(x_{n}-W_{j}(t)\right)
$$

Where, $W_{j}(t+1)$ and $W_{j}(t)$ are defined as the weight vectors after and before updating, respectively, $\eta(t)$ is specified as the learning rate of the adaptation procedure.

The major concern in standard SOM is performance degradation in clustering analysis due to usage of numerous number of classes. While performing with several classes, the class with limited data is neglected due to cluster partition. To overcome this issue an optimal weighting factor is included in the conventional SOM. This optimal weighting factor diminish the number of classes. This class reduction helps to enhance the accuracy of prediction rate. In this scenario, two classes are 
evaluated for weather prediction for examine the rainfall. Likewise, in crop prediction, two classes employed, one class is utilized for rice prediction and another one for ragi prediction. The updated weight equation is illustrated in Eq. (6)

$$
\begin{aligned}
& \quad W_{j}(t+1)=W_{j}(t)+\eta(t)\left(x_{n}-W_{j}(t)\right)+ \\
& \Delta W_{j}(t+1)
\end{aligned}
$$

Here, $\Delta W_{j}$ is chosen based on Learning Vector Quantization (LVQ).

\subsection{Learning vector quantization (LVQ)}

LVQ is a supervised learning method depends on vector quantitation, and also it is a supervised version of SOM for classification purpose. Set initial synaptic weights to small random values, with an interval of $[0,1]$, and allocate a positive value to the learning rate parameter $\alpha=0.9$. The learning rate of alpha is varied from 0.1 to 0.9 and it is found that the maximum efficiency is obtained for alpha value 0.9. So, the learning rate of the parameter $\alpha$ is set to 0.9 .

Initialize the input vector $x_{n}$ from the random data space. If the class labels of $x_{n}$ and the weight vector $W_{j}$ are close means, $W_{j}$ is moved in LVQ in the direction of $x_{n}$. If the class labels of $x_{n}$ and $W_{j}$ are different, $W_{j}$ is moved away from the input vector $x_{n}$. Suppose, the weight vector $W_{j}(t)$ at iteration $t$ is close to the input vector $x_{n}$, the weight vector is mathematically illustrated as follows,

$$
\begin{gathered}
\Delta W_{j}(t)=\arg \min _{j}\left\|x_{n}-W_{j}(t)\right\|_{2}, \\
j=1,2,3 \ldots m
\end{gathered}
$$

Let, the class of $W_{j}(t)$ is denoted as $C W_{j}$ and the class of $x_{n}$ is stated as $C x_{n}$.

The weight vector $\Delta W_{j}(t)$ is adjusted as follows,

If the both classes are similar $C W_{j}=C x_{n}$, Eq. (5) is updated as follows,

$$
\Delta W_{j}(t+1)=W_{j}(t)+\eta(t)\left(x_{n}-W_{j}(t)\right)
$$

If the both classes are different $C W_{j} \neq C x_{n}$, then

$$
\Delta W_{j}(t+1)=W_{j}(t)-\eta(t)\left(x_{n}-W_{j}(t)\right)
$$

Based on the condition, either Eq. (8) or (9) updated in the Weight equation (6), the respective equation determined as an optimal future weight function of SOM. Substitute, the weight function equation (6) in Eq. (2). The output of predicted value is examined in Eq. (10),

$$
y_{n}=y_{n}(p)=f\left(x_{n} \times W_{j}(t+1)\right)
$$

This generated optimal weighting factor helps to enhance the clustering analysis (grouping a set of weights), which is categorized into three ways such as ideal clustering, non-linear clustering and linear clustering. Advanced method (W-SOM) mainly concentrates on linear clustering in Fig. 1, because it requires less number of attributes to examine the clustering. On the other hand, the ideal and non-linear clustering analysis are not shown much concentration, which is displayed in Figs. 2 and 3, because in nonlinear clustering, the collusion between the two classes are extremely high and it requires more of attributes to separate the classes. Likewise, in ideal clustering, the marginal distance between the two classes are very high. This marginal distance degrades the performance evaluation and accuracy of prediction.

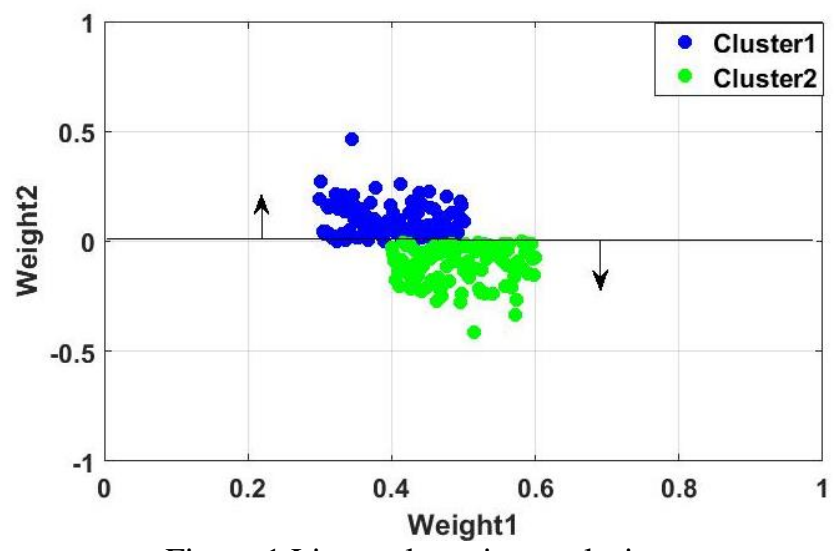

Figure.1 Linear clustering analysis

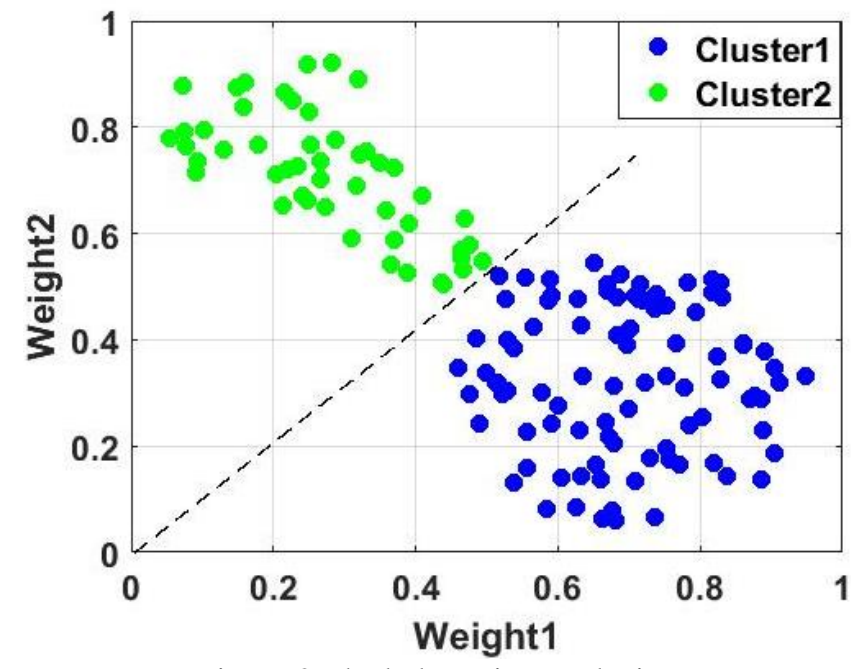

Figure.2 Ideal clustering analysis 


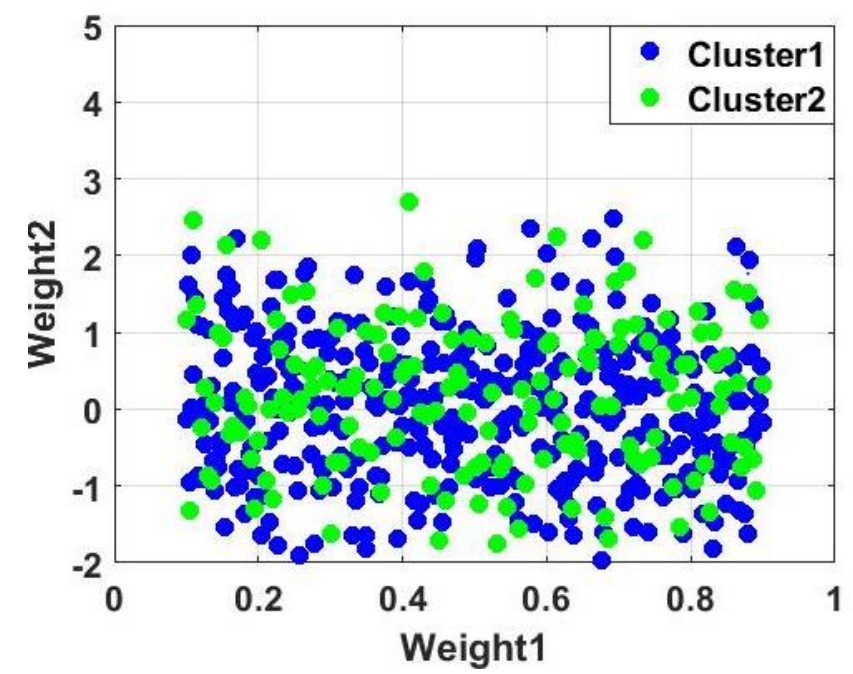

Figure.3 Non-linear clustering analysis

In linear clustering, the clustering efficiency is improved by minimizing the distance between individual weights, which is based on hit point value. Due to this action, the WCE is gradually reduced and the employed weighting factor is capable for organizing large and complex data sets, also eliminates the irrelevant attributes.

\section{Result and discussion}

In this section, the experimental outcome is described in detailed, which was implemented in PC with $1.8 \mathrm{GHz}$ Pentium IV processor using MATLAB (version 6.5). Examination of weather forecasting utilizing the advanced scheme Weighted-SOM (WSOM) algorithm provides enhanced accuracy in weather and crop prediction. In order to estimate the efficiency of the proposed technique, the performance of the proposed approach was compared with the existing methods: KNN, SOM and E-NN methods. H.Y. Kung, T.H. Kuo, C.H. Chen, and P.Y. Tsai, [15] investigated a new system based on the ENN. This system derives the weighted average of all remaining network models to improve the accuracy of the prediction. This experiment was carried out on the database: agriculture and food agency of council of agriculture in Taiwan to validate its result in terms of error rate.

\subsection{Evaluation parameters}

In this advanced scheme for weather forecasting in agriculture is illustrated with a few of the evaluation metrics. This evaluation metrics is employed to evaluate the effectiveness of weather and crop prediction in order to justify theoretical and practical development of this system. Some of the undertaken metrics for evaluation purpose are accuracy, sensitivity and specificity, Mean Absolute Error (MAE) and Mean Absolute Percentage Error (MAPE).

Sensitivity and specificity help to understand the relationships between input and output variables in a system and also testing the robustness of a model in the presence of uncertainty.

$$
\begin{aligned}
& \text { Sensitivity }=\frac{\text { No.of TP }}{\text { No.of } T P+\text { No.of FN }} \times 100 \\
& \text { Specificity }=\frac{\text { No.of } T N}{\text { No.of } T N+\text { No.of FP }} \times 100
\end{aligned}
$$

Accuracy is the appropriate evaluation metric for finding the efficiency of crop prediction and weather prediction.

$$
\text { Accuracy }=\frac{T P+T N}{T P+T N+F P+F N} \times 100
$$

Where, $T P$ is represented as true positive, $T N$ is stated as true negative, FP is denoted as false positive and $F N$ is denoted as false negative.

MAE is a quantity, which is applied to measure the predicted value to the eventual outcomes. Mathematical equation for MAE is illustrated below,

$$
M A E=\frac{1}{N} \sum_{j-1}^{N}\left|y_{n}-x_{n}\right|
$$

MAPE is a measure of prediction accuracy of a forecasting method in statistics, for example in trend estimation, the general equation for MAPE is determined below,

$$
M A P E=100 / N \sum_{j-1}^{N}\left|\frac{y_{n}-x_{n}}{y_{n}}\right|
$$

Where, $y_{n}$ is evaluated as predicted value, $x_{n}$ is determined as true value, $N$ is represented as number of samples.

\subsection{Result of classification evaluation}

Performance evaluation of the advanced method (W-SOM) was estimated based on the metrics like sensitivity, specificity, accuracy, MAE and MAPE. Here, the existing methods: KNN, SOM and E-NN [15] and the advanced scheme (W-SOM) were evaluated in three different combinations of testing and training percentage like $40 \%$ training and $60 \%$ testing, $70 \%$ training and 30\% testing, $80 \%$ training and $20 \%$ testing of collected data, which is displayed in Table 3. 
Table 3. Evaluation measures for existing and proposed method

\begin{tabular}{|c|c|c|c|c|c|c|c|}
\hline Methods & $\begin{array}{c}\text { Training } \\
(\%)\end{array}$ & $\begin{array}{c}\text { Testing } \\
(\%)\end{array}$ & Sensitivity & Specificity & $\begin{array}{c}\text { Accuracy } \\
(\%)\end{array}$ & MAE & MAPE (\%) \\
\hline \multirow{3}{*}{$\mathrm{KNN}$} & $40 \%$ & $60 \%$ & 0.49 & 0.482 & 59.91 & 0.468 & 36.149 \\
\hline & $70 \%$ & $30 \%$ & 0.509 & 0.49 & 62.15 & 0.48 & 35.368 \\
\hline & $80 \%$ & $20 \%$ & 0.32 & 0.43 & 63.87 & 0.466 & 35.113 \\
\hline \multirow{3}{*}{ SOM } & $40 \%$ & $60 \%$ & 0.872 & 0.893 & 63.43 & 0.361 & 17.943 \\
\hline & $70 \%$ & $30 \%$ & 0.907 & 0.88 & 63.92 & 0.356 & 18.02 \\
\hline & $80 \%$ & $20 \%$ & 0.909 & 0.90 & 64.02 & 0.372 & 17.98 \\
\hline \multirow{3}{*}{ ENN [15] } & $40 \%$ & $60 \%$ & 0.881 & 0.88 & 64.07 & 0.360 & 17.956 \\
\hline & $70 \%$ & $30 \%$ & 0.909 & 0.882 & 62.43 & 0.385 & 18.09 \\
\hline & $80 \%$ & $20 \%$ & 0.9 & 0.906 & 65.12 & 0.351 & 17.690 \\
\hline \multirow{3}{*}{$\begin{array}{l}\text { Proposed } \\
\text { W-SOM }\end{array}$} & $40 \%$ & $60 \%$ & 0.89 & 0.90 & 64.58 & 0.356 & 17.893 \\
\hline & $70 \%$ & $30 \%$ & 0.910 & 0.897 & 63.22 & 0.369 & 18.165 \\
\hline & $80 \%$ & $20 \%$ & 0.92 & 0.912 & 65.69 & 0.341 & 17.379 \\
\hline
\end{tabular}

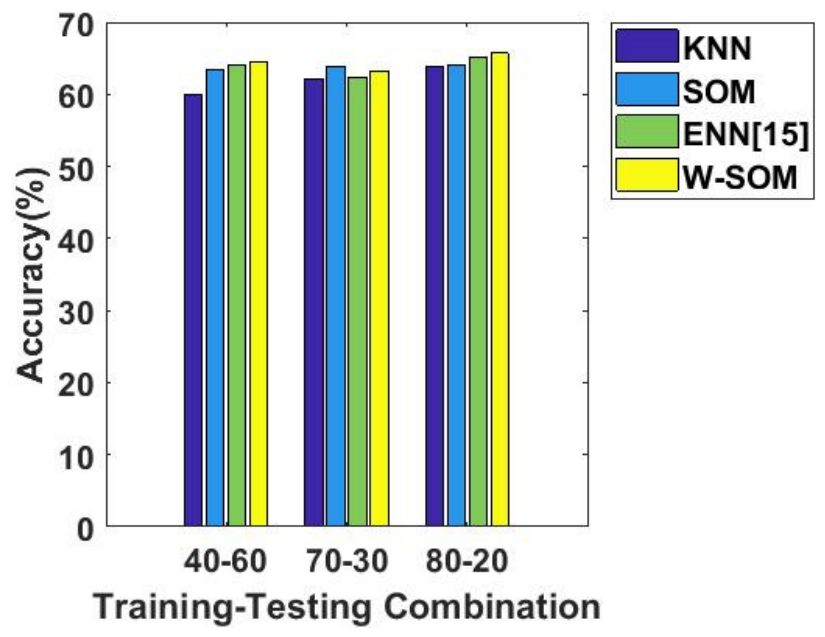

Figure.4 Accuracy graph comparison for advanced and existing scheme

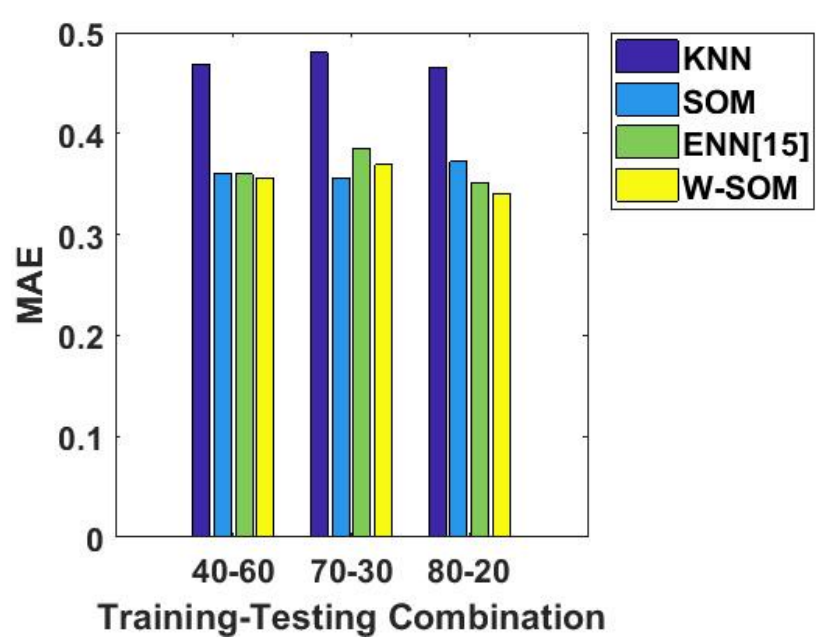

Figure.5 MAE graph comparison for advanced and existing scheme

Table 3 shows the performance evaluation of existing methodologies and the proposed method. In this scenario, W-SOM span the entire multi-

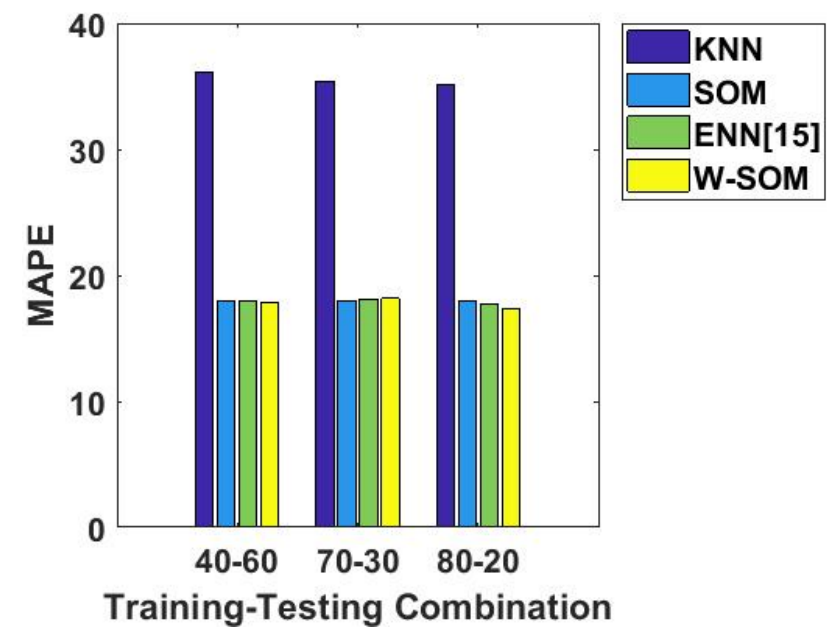

Figure.6 MAPE graph comparison for advanced and existing scheme

dimensional data space and attempt to find points in measurement space that are representative of the data space. Also, W-SOM able to capture the non-linear characteristics of the data and preserve quantitative relationships between the atmosphere and local surface variables. Evaluation metrics confirms that the advanced scheme performs significantly in weather and crop prediction compared to previous methods in terms of accuracy, MAE, MAPE, sensitivity and specificity. Table 3 clearly indicates that the proposed method performs effectively in large dataset.

Fig. 4 specifies that the advanced scheme (WSOM) performs effectively than the existing methodologies K-NN, SOM and ENN. Standardly, the W-SOM scheme shows $65 \%$ of accuracy in both weather and crop prediction for all combination of testing and training percentage.

Fig. 5 determines the MAE graph comparison of existing and advanced scheme in all different combination of testing and training percentage of collected data. Here, the existing methodology K-NN 
shows poor performance in terms of MAE. But, the comparison between SOM, ENN and W-SOM confirms that the advanced scheme performs successfully, and shows 0.31 and 0.10 reduction in MAE associated to SOM and ENN.

Fig. 6 illustrates that the MAPE value of advanced method is superior compared to existing approaches. Gap between the predicted and target value is gradually reduced in Weighted-SOM, which shows $17.8 \%, 0.5 \%$ and $0.3 \%$ reduction in MAPE value associated to KNN, SOM, and ENN schemes.

\section{Conclusion}

This paper evaluated a statistical investigation of weather forecasting that was conducted in the Mysore region. The scope of this experiment was to enhance the rice and ragi productivity in high rainfall regions by examining the accurate rainfall and crop prediction. In this scenario, a weighted-SOM scheme was implemented in order to improve the prediction accuracy. Associated to the other obtainable approaches for weather and crop prediction, the advanced scheme delivered an effective performance by means of accuracy, MAE and MAPE, around 0.5$2 \%$ enhancement than the previous methods. In the future work, for further improving the crop prediction rate, a combination of parallel layer regression along with deep belief network strategy is developed.

\section{References}

[1] A. Mahato, "Climate Change and its Impact on Agriculture", International Journal of Scientific and Research Publications, Vol.4, No.4, pp. 1-6, 2014.

[2] J.L. Hatfield, K.J. Boote, B.A. Kimball, L.H. Ziska, and R.C. Izaurralde, "Climate impacts on agriculture: implications for crop production", Agronomy Journal, Vol.103, No.2, pp.351-370, 2011.

[3] T. Mavromatis, "Spatial resolution effects on crop yield forecasts: An application to rainfed wheat yield in north Greece with CERES-Wheat", Agricultural Systems, Vol.143, pp.38-48, 2016.

[4] L. Hong-ying, H. Yan-lin, Z. Yong-juan, and Z. Hui-ming, "Crop yield forecasted model based on time series techniques", Journal of Northeast Agricultural University (English Edition), Vol.19, No.1, pp.73-77, 2012.

[5] P. Roudier, B. Muller, P. d'Aquino, C. Roncoli, M.A. Soumaré, L. Batté, and B. Sultan, "The role of climate forecasts in smallholder agriculture: lessons from participatory research in two communities in Senegal", Climate Risk Management, Vol.2, pp.42-55, 2014.
[6] S.D. Sawaitul, K.P. Wagh, and P.N. Chatur, "Classification and prediction of future weather by using back propagation algorithm-an approach", International Journal of Emerging Technology and Advanced Engineering, Vol.2, No.1, pp.110-113, 2012.

[7] N. Salot and P.R. Swaminarayan, "A Survey on Rainfall Forecasting using Image Processing Technique", International Journal in IT \& Engineering, Vol.3, No.2, pp.126-132, 2015.

[8] D.K. Bolton and M.A. Friedl, "Forecasting crop yield using remotely sensed vegetation indices and crop phenology metrics", Agricultural and Forest Meteorology, Vol.173, pp.74-84, 2013.

[9] P. Sarlin, "A weighted SOM for classifying data with instance-varying importance", International Journal of Machine Learning and Cybernetics, Vol.5, No.1, pp.101-110, 2014.

[10] V. Aggarwal, A.K. Ahlawat, and B. N. Pandey, "A weight initialization approach for training Self Organizing Maps for clustering applications", In: Proc. of $3^{\text {rd }}$ International Conf. on Advance Computing Conference (IACC), 2013.

[11] P. Bose, N.K. Kasabov, L. Bruzzone, and R.N. Hartono, "Spiking Neural Networks for Crop Yield Estimation Based on Spatiotemporal Analysis of Image Time Series", IEEE Transactions on Geoscience and Remote Sensing, Vol.54, No.11, pp.6563-6573, 2016.

[12] C. Lennard, and G. Hegerl, "Relating changes in synoptic circulation to the surface rainfall response using self-organizing maps", Climate Dynamics, Vol.44, No.3-4, pp.861-879, 2015.

[13] M.C. Morocho, A.V. Ines, W.E. Baethgen, B. Rodríguez-Fonseca, E. Han, and M. RuizRamos, "Crop yield outlooks in the Iberian Peninsula: Connecting seasonal climate forecasts with crop simulation models", Agricultural Systems, Vol.149, pp.75-87, 2016.

[14] O. Satir and S. Berberoglu, "Crop yield prediction under soil salinity using satellite derived vegetation indices", Field Crops Research, Vol.192, pp.134-143, 2016.

[15] H.Y. Kung, T.H. Kuo, C.H. Chen, and P.Y. Tsai, "Accuracy Analysis Mechanism for Agriculture Data Using the Ensemble Neural Network Method", Sustainability, Vol.8, No.8, pp.735, 2016. 